\section{Egg Labeling for Gastric-Emptying Studies}

TO THE EDITOR: We were delighted to read some back-tobasics research in the November JNM and thank Knight et al. (1) for a much-needed evaluation of gastric-emptying labeling efficacy. Mixing a radiopharmaceutical and meal does not guarantee either successful labeling or, indeed, maintenance of the label in vivo. Nonetheless, a variety of different meal compositions have been used with varying success for gastric-emptying studies, including but not limited to chicken liver, pancakes, mushrooms, noodles, eggs (omelet, scrambled, whole eggs, egg whites, commercial egg substitute, and hard-boiled eggs), rice pudding, beef stew, cottage cheese, and porridge (1-3). In Australia, there is anecdotal evidence that solid-phase gastric-emptying studies are also performed using marshmallows, toasted sandwiches, commercially available egg-based breakfast burgers, and even egg-based milk shakes. In recent years, the need for palatability and ease of preparation and, on occasion, the need to address cultural issues appear to have played a role in the emergence of alternative meal types. Unfortunately, many of the alternative meal types have not had reference values validated or indeed the label efficacy determined. Moreover, widespread use of the microwave as a more convenient cooking method may have a significant impact on label integrity; one cannot simply adopt established reference values for pan (griddle)-cooked eggs. We thank Knight et al. (1) for highlighting these issues.

Despite recognition that the use of whole eggs is in widespread practice, gastric-emptying studies have traditionally required egg whites only-in theory, to improve label integrity in vivo (4). Consequently, we were cautious in interpreting the results comparing egg substitute with whole eggs without an account of the performance of egg-white meals. A comparative analysis of other common methods of meal preparation would have strengthened the external validity of this research.

In a recent investigation at Charles Sturt University, comparative analysis was performed on the labeling efficiency of eggs (whites, whole eggs, and precooked commercial breakfast burgers), tofu, marshmallows, and beef (raw meat and precooked commercial breakfast burgers). The method of cooking (precooked, griddle, or microwave) and the time of radiopharmaceutical administration (raw, semicooked, or cooked) were also evaluated. These results (unpublished) showed that the labeling efficiency at $1 \mathrm{~h}$ after incubation in a simulated gastric environment for egg whites $(96.1 \%)$ and whole eggs $(94.8 \%)$ was similar to the labeling efficiency for beef $(97.0 \%)$, whereas poorer efficacy was noted for precooked meat $(83.7 \%)$, precooked eggs $(66.7 \%)$, tofu $(64.4 \%)$, and marshmallows $(29.6 \%)$. The tofu was confounded by a large disparity in results between griddle-cooked tofu $(81.3 \%)$ and microwave-cooked tofu $(47.5 \%)$. It mattered naught whether the radiopharmaceutical was added to the raw $(96.2 \%)$ or semicooked $(96.0 \%)$ meal; however, addition to precooked food performed poorly $(75.1 \%)$. Similarly, whether a griddle $(96.1 \%)$ or a microwave $(96.2 \%)$ was used as the method of cooking was inconsequential, after tofu was excluded.

COPYRIGHT @ 2008 by the Society of Nuclear Medicine, Inc.
The results from Charles Sturt University support the use of egg substitute as an alternative to both egg whites and whole eggs, although the findings for whole eggs between microwave and griddle cooking are discordant with those of Knight et al. (1). In the interest of maintaining scintigraphy as the gold standard for gastricemptying evaluations, we hope that practitioners use informed decision making before adopting an alternative, nonvalidated meal type.

\section{REFERENCES}

1. Knight LC, Kantor S, Doma S, Parkman HP, Maurer AH. Egg labeling methods for gastric emptying scintigraphy are not equivalent in producing a stable solid meal. J Nucl Med. 2007;48:1897-1900.

2. Donohoe KJ, Maurer AH, Ziessman HA, Urbain J-LC, Royal HD, Martin-Comin J. Society of Nuclear Medicine Procedure Guideline for Gastric Emptying and Motility. Reston, VA: Society of Nuclear Medicine; 2004. Version 2.0.

3. Kwiatek MA, Jones KL, Burch WM, Horowitz M, Dylan FD. Use of Technegas as a radiopharmaceutical for the measurement of gastric emptying. Eur J Nucl Med. 1999;26:903-906.

4. Knight LC, Fisher RS, Malmud LS. Comparison of solid food markers in gastric emptying studies. In: Raynaud C, ed. Nuclear Medicine and Biology Advances. Vol III. Paris, France: Pergamon Press; 1982:2407-2410.

\author{
Geoffrey M. Currie \\ Janelle M. Wheat \\ Charles Sturt University \\ Wagga Wagga, Australia
}

DOI: $10.2967 /$ jnumed.107.050385

REPLY: We appreciate the comments by Drs. Currie and Wheat on our study that evaluated different methods to prepare an eggbased radiolabeled meal for gastric-emptying scintigraphy (1). The message of our study was that labeling methods and cooking methods need to be evaluated for any solid meal before one can adopt it for performing a gastric-emptying study. We also demonstrated in our paper that in vitro evaluation of stability in "simulated gastric conditions" may give different results if the incubation solution is dilute hydrochloric acid rather than human gastric fluid, which would contain pepsin in addition to acid. In attempting to directly answer Drs. Currie and Wheat, we find that the data mentioned in their letter are brief and omit adequate details on how their foods were combined with radiotracers and how cooking, digestion, and quantification were performed. Our studies support using a low-fat egg-substitute meal (EggBeaters; ConAgra Foods, Inc.), which is the meal suggested by a consensus working group of the Society of Nuclear Medicine and the American Neurogastroenterology and Motility Society (2). We agree on the importance of developing alternative meals and would urge the researchers at Charles Sturt University to proceed with full publication of the details of their studies.

\section{REFERENCES}

1. Knight LC, Kantor S, Doma S, Parkman HP, Maurer AH. Egg labeling methods for gastric emptying scintigraphy are not equivalent in producing a stable solid meal. J Nucl Med. 2007;48:1897-1900.

2. Abell TL, Camilleri M, Donohoe K, et al. Consensus recommendations for gastric emptying scintigraphy: a joint report of the American Neurogastroenterology and 
Motility Society and the Society of Nuclear Medicine. Am J Gastroenterol. November 19, 2008 [Epub ahead of print].

\author{
Linda C. Knight \\ Henry P. Parkman \\ Alan H. Maurer \\ Temple University School of Medicine \\ Philadelphia, Pennsylvania
}

DOI: $10.2967 /$ jnumed.108.050443

\section{REFERENCE}

1. Freeman LM. Don't bury the V/Q scan: it's as good as multidetector CT angiograms with a lot less radiation exposure. J Nucl Med. 2008;49:5-8.

\section{Bob Bury \\ Leeds Teaching Hospitals Trust Leeds, United Kingdom}

DOI: 10.2967/jnumed.108.050419

\section{Scintigraphy or Multidetector CT Angiography for Suspected Pulmonary Embolism?}

TO THE EDITOR: Leonard Freeman's perspective on the use of the ventilation-perfusion scan (1) will be a useful resource for those of us struggling to educate our clinicians on the relative utility of scintigraphy and multidetector CT angiography in the diagnosis of pulmonary embolism. Like him, we instituted triage using the clinical history and the chest radiograph findings to determine which modality to use. Those with abnormal radiograph findings or a clinical history of significant preexisting cardiopulmonary disease proceed directly to multidetector CT angiography. In the $3 \mathrm{y}$ or so since the introduction of this system, we have seen a significant reduction in the number of indeterminate results with scintigraphy, but some patients still end up getting the wrong investigation, often as a result of insufficient clinical information being available to the radiologist. We are attempting to remedy this problem by using a specific request card for the investigation of suspected pulmonary embolism, and this remedy is currently being piloted.

I note that Dr. Freeman specifically mentions the presence of fluid, atelectasis, and consolidation on the chest radiograph as disqualifying factors for scintigraphy. As he states, this type of gross change is relatively unusual, and in my experience, indeterminate ventilation-perfusion findings are most frequently the result of preexisting chronic obstructive pulmonary disease. These patients often have chest radiograph findings that are relatively normal, with the only changes being some overinflation and a subtle increase in bronchovascular markings. On the occasions when I have gone ahead with a ventilation-perfusion scan slightly against my better judgment because the chest radiograph showed only minor changes of chronic obstructive pulmonary disease, I have nearly always regretted it, the resulting images showing widespread matched defects of ventilation and perfusion. If the vetting process is delegated to radiologists not directly involved in the pulmonary embolism imaging service, it is important that they realize that consolidation, atelectasis, and effusions are not the only findings that make patients unsuitable for scintigraphy, or these radiologists may give the all clear to chest radiographs showing quite marked changes of chronic obstructive pulmonary disease.
REPLY: I am most appreciative of Dr. Bury's kind comments about my views on the use of ventilation-perfusion scintigraphy versus multidetector CT angiography for the study of pulmonary embolic disease. It appears as if we are in accord on most issues; most importantly, the overuse of CT angiography with its associated excessive radiation exposure, particularly to the female breast. The use of the plain chest radiograph for determining which modality to apply has worked quite well.

The one area where we appear to have some difference is how to handle the patient with chronic obstructive pulmonary disease who may have only minimal radiographic changes of "overinflation and a subtle increase in bronchovascular markings." The frequent ventilation-perfusion scintigraphy result of multiple matched defects that troubles Dr. Bury has not been a problem for us. This combination of findings clearly falls into the low-probability category. To my knowledge, we have not had any problem or adverse feedback from our clinicians in calling these studies low probability. An ongoing retrospective review of over 1,200 ventilation-perfusion studies that were performed in 2007 shows that we gave an "intermediate" or "indeterminate" interpretation in less than $5 \%$ of our examinations.

I would like to refer Dr. Bury and other interested parties to a recent prospective comparison of ventilation-perfusion scintigraphy and CT angiography by Anderson et al. that showed a similar outcome for the 2 procedures (1). This comparison should also be of great assistance to nuclear medicine physicians and radiologists who are trying to convince their clinicians to cut down on the excessive use of CT angiography in studying patients with suspected pulmonary embolic disease.

\section{REFERENCE}

1. Anderson DR, Kahn SR, Rodger MA, et al. Computed tomographic pulmonary angiography vs ventilation-perfusion lung scanning in patients with suspected pulmonary embolism: a randomized controlled trial. JAMA. 2007;298:2743-2753.

\author{
Leonard M. Freeman \\ Montefiore Medical Center \\ Bronx, New York
}

DOI: 10.2967/jnumed.108.050450 